\title{
References
}

Barth, W. and Barth, S. (1991). Programme für korrekte Schachendspiele und deren Validierung. Institutsbericht Nr. 34, Institut für Computergraphik, Technische Universität Wien.

Barth, W. and Barth, S. (1992). Validating a Range of Endgame Programs. ICCA Journal, Vol. 15, No. 3, pp. 132-139.

\section{ARTICLES PUBLISHED ELSEWHERE}

\section{THE MULTI-PLAYER VERSION OF MINIMAX DISPLAYS GAME-TREE PATHOLOGY}

D. Mutchler (1993). The multi-player version of minimax displays game-tree pathology (Research Note), Artificial Intelligence, Vol. 64, pp. 323-336.

We reproduce the abstract:

"It is widely believed that by searching deeper in the game tree, the decision maker is more likely to make a better decision. Dana Nau and others have discovered pathology theorems that show the opposite: searching deeper in the game tree causes the quality of the ultimate decision to become worse, not better. The models for these theorems assume that the search procedure is minimax and the games are two-player zero-sum. This report extends Nau's pathology theorem to multi-player game trees searched with maxn, the multi-player version of minimax. Thus two-player zero-sum game trees and multi-player trees are shown to have an important feature in common."

\section{PROOF-NUMBER SEARCH}

L.V. Allis, M. van der Meulen, and H.J. van den Herik (1994). Proof-number search. Artificial Intelligence, Vol. 66, pp. 91-124.

We reproduce the abstract:

"Proof-number search (pn-search) is designed for finding the game-theoretical value in game trees. It is based on ideas derived from conspiracy-number search and its variants, such as applied cn-search and $\alpha \beta-c n$ search. While in cn-search the purpose is to continue searching until it is unlikely that the minimax value of the root will change, pn-search aims at proving the true value of the root. Therefore, pn-search does not consider interim minimax values.

Pn-search selects the next node to be expanded using two criteria: the potential range of subtree values and the number of nodes which must conspire to prove or disprove that range of potential values. These two criteria enable pn-search to treat efficiently game trees with a non-uniform branching factor.

It is shown that in non-uniform trees pn-search outperforms other types of search, such as $\alpha-\beta$ iterative deepening search, even when enhanced with transposition tables, move ordering for the full principal variation, etc. Pn-search has been used to establish the game-theoretical values of Connect-Four, Qubic, and Go-Moku. There pn-search was able to find a forced win for the player to move first. The experiments described here are in the domain of Awari, a game which has not yet been solved. The experiments are repeatable for other games with a non-uniform branching factor.

This article describes the underlying principles of pn-search, presents an appropriate implementation, and provides an analysis of its strengths and weaknesses." 